Научная статья

УДК 338.23

DOI: $10.17213 / 2075-2067-2021-6-170-186$

\title{
НОВЫЕ ПОДХОДЫ К УСТОЙЧИВОСТИ ПРОЕКТОВ МИНЕРАЛЬНО-СЫРЬЕВОГО КОМПЛЕКСА В УСЛОВИЯХ СОВРЕМЕННЫХ ВЫЗОВОВ
}

\author{
Диана Михайловна Дмитриева ${ }^{1}$, \\ Виктория Максимовна Соловьева ${ }^{2 \bowtie}$, Евгения Григорьевна Рутенко ${ }^{3}$ \\ 1, 2, 3 Санкт-Петербургский горный университет, Санкт-Петербург, Россия \\ Idiana-dmitrieva@mail.ru, ОRCID: 0000-0002-4208-4842, AuthorID РИНЦ: 617505 \\ 2vikasolovyova9@gmail.com ${ }^{\bowtie}$, ORCID: 0000-0003-2696-8143, AuthorID РИНЦ: 928987 \\ 3evgewe@mail.ru, AuthorID РИНЦ: 1079584
}

Аннотация. Целью исследования является формирование концептуальных подходов $\kappa$ устойчивости проектов минерально-сырьевого комплекса в условиях повышения уровня турбулентности макроокружения, формирования новых вызовов и тенденичий.

Методологическая основа исследования. Теоретической базой проводимого исследования выступают основы концепчии устойчивости и устойчивого развития, ключевых принципов и особенностей устойчивого развития, концептуальных подходов к стратегической устойчивости. Для определения современных вызовов реализачии проектов в сфере минерально-сырьевых комплексов проведен контент-анализ российской и зарубежной литературы, задействованы аналитические и статистические данные. В работе использованы общенаучные методы анализа, синтеза, обобщения, декомпозищии факторов, элементы стратегического анализа и планирования.

Результаты исследования. Результаты работы состоят в уточнении теоретических аспектов устойчивого развития и устойчивости проектов, в обосновании высокого уровня неопределенности макроэкономических параметров реализации проектов в сфере минерально-сырьевых комплексов ввиду значительной турбулентности внешних факторов и появления новых вызовов, в установлении необходимости трансформации устоявшихся подходов к устойчивому развитию проектов. В исследовании разработаны концептуальные основы стратегической устойчивости проектов, соответствующие новому подходу $\kappa$ оченке устойчивого развития и построенные на учете фактора неопределенности и высокого уровня турбулентности макроокружения.

Перспективы исследования заключаются в практическом использовании разработанных подходов для формирования моделей устойчивой реализаџии проектов в сфере минерально-сырьевых комплексов.

Ключевые слова: минерально-сырьевой комплекс, проекты, устойчивое развитие, новые подходы, вызовы, турбулентность

Для цитирования: Дмитриева Д. М., Соловьева В.М., Рутенко Е.Г. Новые подходы к устойчивости проектов минерально-сырьевого комплекса в условиях современных вызовов// Вестник Южно-Российского государственного технического университета. Серия: Социально-экономические науки. 2021. T. 14, № 6. C. 170-186. http://dx.doi.org/10.17213/20752067-2021-6-170-186.

(ㄱ Дмитриева Д. М., Соловьева В.М., Рутенко Е.Г., 2021 
Благодарности. Исследование выполнено при финансовой поддержкке гранта Президента РФ по государственной поддержкке ведущих научных икол Российской Федерации, проект НШ-2692.2020.5 «Моделирование эколого-сбалансированного и экономически устойчивого освоения углеводородных ресурсов Арктики».

Original article

\title{
NOVEL APPROACHES TO THE MINING PROJECTS'SUSTAINABILITY IN A VIEW OF CURRENT CHALLENGES
}

\author{
Diana M. Dmitrieva ${ }^{1}$, Victoria M. Solovyova ${ }^{2 \bowtie}$, Evgeniya G. Rutenko $^{3}$ \\ 1, 2, ${ }^{3}$ Saint Petersburg Mining University, Saint Petersburg, Russia \\ Idiana-dmitrieva@mail.ru, ORCID: 0000-0002-4208-4842, AuthorID RSCI: 617505 \\ ${ }^{2}$ vikasolovyova9@gmail.com ${ }^{\bowtie}$,ORCID:0000-0003-2696-8143, AuthorID RSCI: 928987 \\ ${ }^{3}$ evgewe@mail.ru, AuthorID RSCI: 1079584
}

\begin{abstract}
The purpose of the research is to create conceptual approaches towards the mining projects' sustainability in a view of an increase in macroeconomic turbulence, appearance of new challenges and trends.

The methodological basis. The theoretical basis of this research consists of the concept of sustainability and sustainable development, key principles and features of sustainable development, conceptual approaches to strategic sustainability. To determine the current challenges of implementing mining projects, a content analysis of Russian and foreign literature was carried out, analytical and statistical data was used. The investigation applied general scientific methods of analysis, synthesis, generalization, decomposition, elements of strategic analysis and planning.

Research result. The results of the study are to clarify the theoretical aspects of sustainable development and sustainability of the investment projects, to substantiate the high level of uncertainty in the macroeconomic parameters of the projects' implementation due to the high level of macroeconomic turbulence and the emergence of new challenges, to determine the need to transform the established approaches to projects'sustainable development. The investigation has developed a conceptual framework for the projects' strategic sustainability, corresponding to a new approach to assessing sustainable development and based on the uncertainty and high level of turbulence in the macroenvironment.

The prospects of the research consist in the practical use of the developed approaches for the formation of models for the sustainable implementation of the mining projects.

Keywords: mineral resource complex, projects, sustainable development, novel approaches, challenges, turbulence

For citation: Dmitrieva D.M., Solovyova V.M., Rutenko E. G. Novel approaches to the mining projects'sustainability in a view of current challenges // Bulletin of the South Russian State Technical University. Series: Socio-economic Sciences. 2021; 14(6): 170-186. (In Russ.). http:// dx.doi.org/10.17213/2075-2067-2021-6-170-186.

Acknowledgments. The research was carried out with the financial support of the grant of the President of the Russian Federation for state support of leading scientific schools of the Russian Federation, the project NSch-2692.2020.5 "Modeling of ecologically balanced and economically sustainable development of hydrocarbon resources of the Arctic».
\end{abstract}


Введение. В настоящее время проблематика устойчивого развития проектов (систем, комплексов) является особенно актуальной - ужесточаются экологические стандарты, меняются подходы и требования к финансированию проектов, интенсивно развивается сектор «зеленой» экономики и энергетики, меняются потребности общества. Осуществление инвестиционных проектов рассматривается не только с позиции получения финансово-экономических показателей, но и в контексте их вклада в достижение глобальных Целей устойчивого развития (ЦУР) [9].

Для проектов в сфере промышленного освоения минерально-сырьевого комплекса (МСК) тематика устойчивости приобретает особую значимость [10-12]. Масштабность таких проектов предопределяет существенные экологические и социальные последствия их реализации, что обуславливает необходимость учета множества факторов при принятии управленческих решений об их осуществлении и разработка стратегий долгосрочного развития. Более того, современные макроэкономические условия свидетельствуют о высокой степени неопределенности и турбулентности макроокружения [5]. Новые тенденции требуют от компаний гибкости и способности не только быстро адаптироваться, но и сохранять устойчивость в долгосрочной перспективе.

Возникающие тенденции на мировых рынках, высокая степень изменчивости окружения бизнеса, бурное развитие технологий, смещение акцента в сторону экологизации производств, повышение значимости социальной составляющей - все это, дополняясь геополитическими и макроэкономическими параметрами, объективно требует пересмотра сложившихся подходов к обеспечению устойчивости реализуемых проектов в сфере МСК, что обуславливает актуальность и значимость предлагаемого исследования.

Теоретические основы устойчивости и устойчивого развития (УР). Согласно классической трактовке устойчивое развитие - это тип развития (системы, комплекса, проекта), предусматривающий учет и согласованность трех ключевых составляющих: экологической, экономической и социальной [16]. Сам термин «устойчивое развитие» впервые был введен еще в 1987 году в докладе «Наше общее будущее», подготовленном Всемирной комиссией $\mathrm{OOH}$ по окружающей среде и развитию [28]. Впоследствии по мере повышения интереса к решению социальных и экологических задач была сформирована целостная концепция устойчивого развития, согласно которой экономический рост не может быть единственным приоритетом при принятии управленческих решений.

Несмотря на общепризнанность основ концепции устойчивого развития, существуют различные подходы к ее интерпретации. В зарубежной литературе понятие устойчивости тесно связывают с социально-экологическими параметрами. В работе зарубежного ученого Дж. Хакла выделено два ключевых подхода к определению устойчивости: а)реформистский подход, согласно которому экономические, экологические и социальные приоритеты должны осуществляться сбалансированно; б) радикальный подход, предполагающий развитие экономических и социальных приоритетов в рамках устанавливаемых экологических ограничений (рис. 1) [29].

Исходя из радикального подхода, можно выделить две категории, лежащие в основе концепции устойчивого развития: с одной стороны, потребности общества (в том числе приоритетные потребности), с другой - ограничения, связанные с социально-экологическими параметрами [29]. Именно в рамках данной концепции был сформирован ряд подходов к оценке стоимости природных ресурсов (в контексте признания исчерпаемости ресурсного потенциала).

Вопреки тому факту, что концепция устойчивого развития является общепринятой, существует мнение о том, что «устойчивость» и «развитие» являются несопоставимыми понятиями. Это связано с тем, что «развитие» непременно сопряжено с движением и динамичными переменами. Если же обратиться к термину «устойчивость», то можно сказать, что под ним понимают способность системы к сохранению и поддержанию определенных характеристик во времени [25; 30]. Противоречие связано и с тем, что в научной литературе продолжаются дискуссии относительно того, являются ли понятия «устойчивое развитие» и «устойчивость» одинаковыми или все же имеют различную смысловую нагрузку [30]. 
Согласно определению UNESCO устойчивость - это общая парадигма будущего развития и устройства мира на основе триединства экономики, экологии и социума, в то время как устойчивое развитие - это конкретные шаги и направления, реализуемые для ее достижения ${ }^{1}$. Однако выдвигаются и предположения о том, что обозначенные концепции не только не имеют ничего общего, но даже противоречат друг другу [25].

Как экономическая категория «устойчивость» отражает «особое состояние» хозяйственной системы (проекта, комплекса), гарантирующее возможность ее эффективного развития в условиях высокой степени нестабильности макроокружения [16]. Соответственно, в зависимости от факторов, влияющих на рассматриваемую систему, выделяют финансовую, ценовую, рыночную и другие виды устойчивости.

В экономической теории устойчивость рассматривается с позиции концепции экономического равновесия, согласно которой достижение равновесного состояния системы является одной из важнейших макроэкономических задач [20]. В финансовом менеджмен-

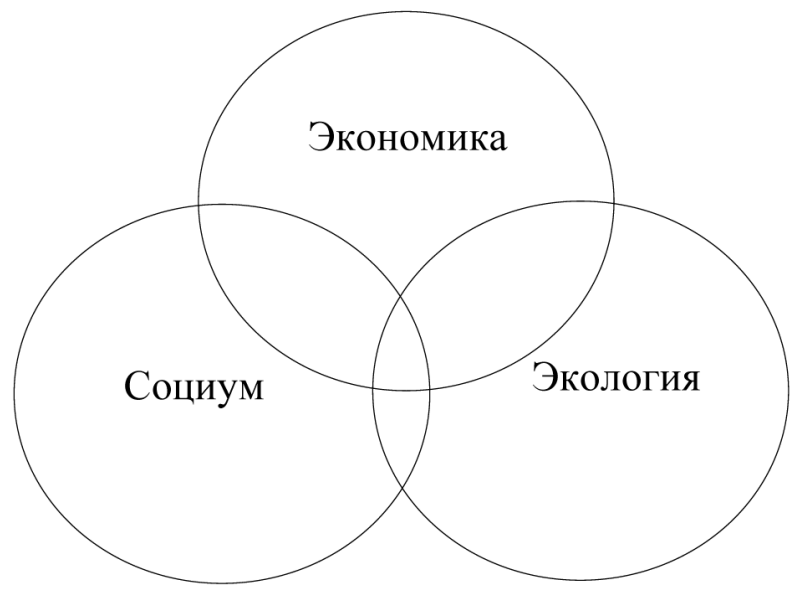

a) те же данный термин связывают с достижением необходимых финансово-экономических показателей (в пределах установленных требований и норм) [17].

Среди основных признаков, характеризующих устойчивость, выделяют удовлетворение текущих потребностей без ущерба для будущих поколений, рациональное использование имеющегося ресурсного потенциала, учет общественных интересов и экологических аспектов. В некоторых источниках литературы устойчивость рассматривается исключительно с позиции охраны окружающей среды (environmental sustainability) ${ }^{2}$ [29].

В настоящее время по мере усложнения параметров макросреды и повышения требований и стандартов, предъявляемых к компаниям и проектам, содержательные аспекты устойчивости постепенно расширяются. Так, в зарубежной практике свое применение нашел новый подход к инвестированию - «ответственное инвестирование», в основе которого лежит учет факторов управления ESG (Ecological, social \& governmental) [27].

Более того, теперь реализация проектов и деятельность компаний рассматриваются

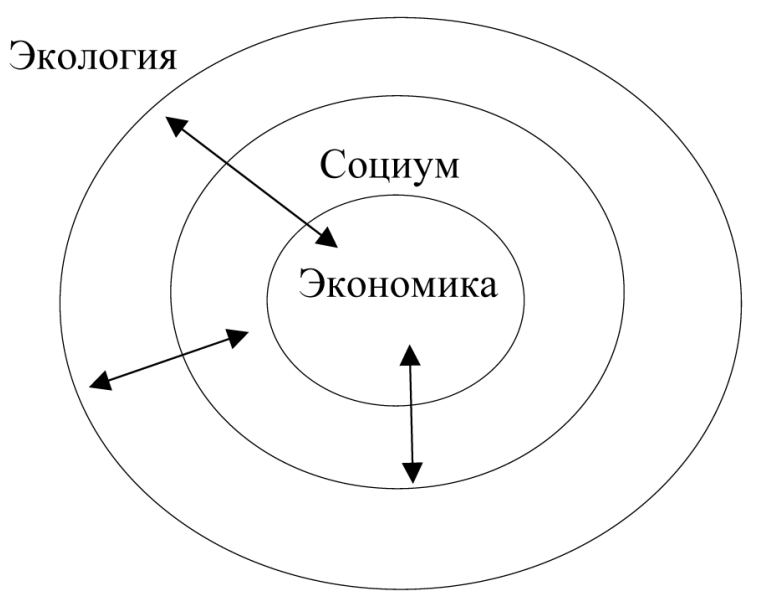

б)

Рис. 1. Различные подходы к интерпретации устойчивости и устойчивого развития:

a) реформистский подход; б) радикальный подход [29]

Fig. 1. Various approaches to the interpretation of sustainability and sustainable development:

a) reformist approach; b) radical approach [29]

1 United Nations General Assembly «Sustainable development: managing and protecting our common environment 2005». World Summit Outcome, 2005. [Electronic resource]. URL: http://daccess-ddsny.un.org/doc/UNDOC/GEN/ N05/487/60/PDF/N0548760.pdf (date accessed: 27.09.2021).

2 Environmental Sustainability [Electronic resource] // Advances in Ecological Research, 2019. URL: https://www. sciencedirect.com/topics/agricultural-and-biological sciences/environmental-sustainability (date accessed: 23.09.2021). 
не только в контексте достижения показателей устойчивого развития (УР) на микроуровне, но и с позиции возможности их вклада в достижение глобальных Целей УР, направленность которых выходит за рамки классической триады «экономика-экология-общество» [4].

Для минерально-сырьевого комплекса проблематика обеспечения устойчивости реализации проектов представляется особенно актуальной. С одной стороны, указанный сектор формирует значительный вклад в развитие экономики, с другой стороны, его функционирование связано с повышенными экологическими и социальными рисками [12]. В зарубежной научной литературе выдвигается тезис о том, что ведение горных работ априори не является устойчивым ввиду необратимости формируемых последствий.

В то же время зарубежный ученый Дж. Найн высказал идею о том, что хоть промышленное освоение минерально-сырьевого сектора и неустойчиво, но его УР может внести свой вклад в достижение заявленных ранее глобальных целей устойчивого развития ${ }^{3}$. Очевидно, что добыча и переработка полезных ископаемых неизбежны ввиду высокой значимости природных ресурсов для экономики и промышленности всех стран мира. Поэтому сегодня фокус смещается в сторону того, как проектам и компаниям, осуществляющим деятельность в секторе МСК, адаптироваться к возникающим тенденциям (низкоуглеродное развитие, «зеленая» экономика) и новым вызовам, а также как обеспечить устойчивый рост в долгосрочной перспективе, опираясь на триединство ключевых составляющих ${ }^{4}$.

Ключевые вызовы, формирующие неопределенность реализации проектов в сфере МСК. В последние годы сектор МСК претерпевает существенные преобразования, связанные с возникающими тенденциями на глобальном рынке сырьевых ресурсов: меняется структура потребностей, повышаются требования и стандарты, возрастает роль цифровизации и пр. [2; $6 ; 8 ; 31]$.
B отчете Deloitte «Тенденции развития-2021» было выделено десять новых трендов, оказывающих непосредственное влияние на функционирование мирового сектора освоения минеральных ресурсов, среди которых выделяют усиление процессов декарбонизации, повышение значимости использования альтернативных источников энергии, изменение ключевых принципов инвестирования [18]. В обозначенном отчете выдвинут тезис о том, что для компаний важный «урок» пандемийного и пост-пандемийного периодов (COVID-19) состоит в осознании ценности работы по укреплению устойчивости в постоянно меняющихся, турбулентных условиях.

Понятие «турбулентности» было введено в науку И. Ансоффом. Данный термин характеризует динамизм среды, оцениваемый такими параметрами, как степень непостоянства рыночной среды, скорость изменений, интенсивность конкуренции и появление новых технологических возможностей [22]. Чем выше уровень турбулентности макроокружения, тем более непредсказуемы последствия преобразований. В предыдущих исследованиях авторами статьи было установлено, что для современных отраслей МСК, в частности для энергетического сектора, характерен значительный уровень турбулентности (наиболее высокий по классификации И. Ансоффа), чему есть объективное обоснование [5]. В качестве ключевых трендов, оказывающих воздействие на скорость преобразований на уровне мирового рынка энергоресурсов, следует выделить [5; 18]:

- усложнение технологической составляющей под влиянием таких параметров, как истощение традиционных запасов углеводородного сырья, ухудшение горно-геологических условий введенных в эксплуатацию месторождений;

- усиление процессов цифровизации и дигитализации, требующих трансформации не только производственных процессов, но и изменения подходов к управлению;

3 Keen J. Can be mining sustainable? [Electronic resource]. URL: https://ensia.com/articles/mine-over-matter/ (date accessed: 15.09.2021).

4 The Importance of Sustainability in Mining Operations. Engineers Australia [Electronic resource]. URL: https:// www.engineersaustralia.org.au/News/importance-sustainability-mining-operations (date accessed: 17.09.2021). 
- ужесточение конкурентной борьбы на традиционных рынках углеводородных ресурсов, определяющее ценность и роль формирования конкурентных преимуществ для удержания позиции на рынке нефтепродуктов;

- интенсификация тенденций «зеленого» роста, определяющих высокие экологические стандарты и требования, предъявляемые к проектам и компаниям в сфере добычи углеводородных ресурсов;

- повышение роли социальных аспектов, необходимость проведения открытой политики и учета интересов ключевых стейкхолдеров, в частности общества.

Последние события, связанные с глобальной пандемией (COVID-19), предопределили повышение степени неопределенности и уровня турбулентности на ключевых рынках природных ресурсов. Так, обращаясь к глобальному энергетическому сектору, стоит заключить, что пандемия внесла значительные коррективы в представление о его будущем функционировании и развитии [7].

Последствия ограничительных мер нашли свое отражение в резком спаде экономической активности на глобальном рынке нефтепродуктов и ужесточении ценовой конкуренции среди основных производителей энергоресурсов. По оценкам Междуна- родного энергетического агентства (МЭА), в 2020 году мировой спрос на нефть снизился в среднем на $10 \%$ в сравнении с аналогичными показателями 2019 года, что отразилось и на структуре потребления первичной энергии (рис. 2).

Раньше прогнозы цены на нефть отличались «оптимистичностью»: так, по прогнозу The International Energy agency (IEA), составленному в 2019 году, цена на нефть будет непрерывно расти и к 2029-2030 годам достигнет значения в 110 долл./барр. ${ }^{5}$ Однако в 2020 году прогнозные оценки изменили вектор. Так, компания Eni, лидер на мировом рынке нефтегазовых ресурсов, прогнозирует медленный рост цен на нефть за счет постепенного восстановления мирового рынка энергоресурсов — к 2030 году цена не поднимется выше 70 долл./барр. ${ }^{6}$ Это мнение разделяют не только нефтегазовые компании (BP, Shell), но и международные агентства и организации (Deloitte, IMF, IEA и др.) [24].

На фоне высокой степени неопределенности энергетического сектора начала постепенно пересматриваться роль ископаемого топлива в структуре спроса на источники энергии. По мнению экспертов Всемирного энергетического совета (WEC), негативные тенденции глобального спроса на нефть сфор-

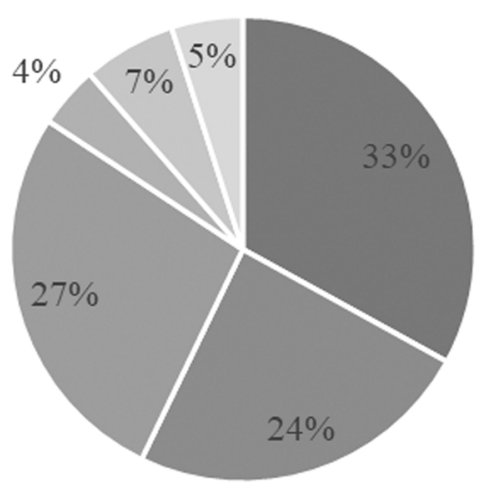

$2019 \Gamma$.

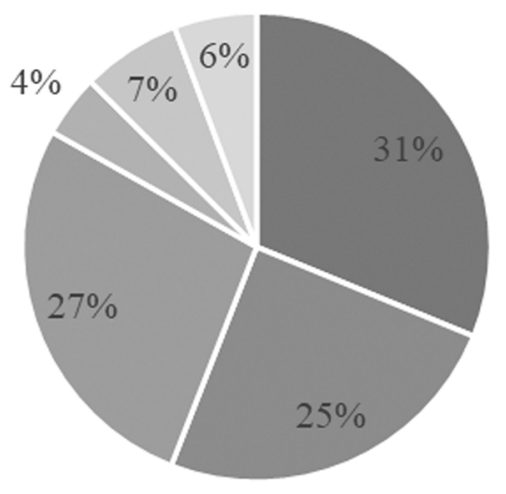

" Нефть

- Природный газ

- Уголь

- Атомная энергия

" Гидроэнергия

Возобновляемые источники ресурсов

Рис. 2. Структура баланса потребления первичной энергии в мире по источникам ${ }^{7}, \%$ Fig. 2. The structure of the balance of primary energy consumption in the world by sources, $\%$

5 Oil-2020 [Electronic resource] // IEA. URL: https://www.iea.org/reports/oil-2020 (date accessed: 15.09.2021).

6 Crude Oil Price Forecast: 2020, 2021 and Long Term to 2030 [Electronic resource]. URL: https://knoema.ru/ infographics/yxptpab/crude-oil-price-forecast-long-term-2016-to-2025-data-and-charts (date accessed: 18.09.2021).

7 Statistical Review of World Energy 2020. 69th edition [Electronic resource] // BP. 2020. 68 p. URL: https://www. bp.com/content/dam/bp/business-sites/en/global/corporate/pdfs/energy-economics/statistical-review/bp-stats-review2020-full-report.pdf. 
мировали новые возможности для компаний и проектов в контексте перераспределения капитала в сторону цифровых решений и экологически чистых технологий ${ }^{8}$. Международное рейтинговое агентство Moody's выдвинуло тезис о том, что именно пандемия и ее последствия стали ключевым «драйвером» к интенсификации усилий по осуществлению глобального энергетического перехода и изменению курса на низкоуглеродный тип развития 9 .

Тренды глобального энергетического перехода предопределяют смещение акцента с ископаемых источников получения энергии к возобновляемым, ввиду чего, по прогнозам международных экспертов, спрос на нефть в ближайшем будущем будет снижаться, в то время как существенно возрастут потребности в металлах, требуемых для создания «зеленых» технологий ${ }^{10}$ [23]. Согласно сценариям ВР, отраженным в «The Energy Outlook», к 2050 году структура энергобаланса кардинально изменится в пользу превалирующей доли возобновляемых источников энергии ${ }^{11}$. Сама компания стремится диверсифицировать собственные производственные мощности. По данным разработанной и утвержденной в 2020 году Стратегии, ВР планирует сократить добычу нефти на собственных активах более чем на $40 \%$ и приостановить целый ряд перспективных проектов по освоению углеводородного сырья.

По прогнозу DNV GL Energy transition outlook 2020, доля нефти и угля в структуре предложения энергии в мире будет постепенно сокращаться. И если в 2018 году удельный вес нефти в общей структуре мирового предложения составлял $29 \%$, а угля - $27 \%$, то к 2040 году ситуация должна кардинально измениться - доля нефти составит не более $20 \%$, а угля — лишь 15,5\% [26].
Тенденции «зеленого» роста оказывают влияние и на финансирование новых нефтегазовых проектов. Так, в 2021 году крупнейшие банки США (UBS bank, Multiple United States banks, Wells Fargo \& Company, Goldman Sachs) заявили о приостановке инвестирования шельфовых проектов, связанных с добычей нефтяных ресурсов ${ }^{12}$. Комментируя сложившуюся ситуацию, компания Deloitte выделила два пути устойчивого развития нефтегазового сектора в новых условиях: 1) диверсификация бизнеса в пользу создания возобновляемых источников энергии и 2) внедрение прогрессивных технологий, при которых выбросы загрязняющих веществ и иные экологические последствия будут сводиться нулю ${ }^{13}$.

На рисунке 3 систематизированы ключевые вызовы энергетического сектора, определяющие высокий уровень турбулентности макроокружения.

Важно заключить, что по мере роста неопределенности макроокружения и интенсификации динамики тенденций на глобальных рынках возникает объективная необходимость пересмотра существующих подходов к УР. Управленческие решения в отношении развития компаний и реализации инвестиционных проектов приобретают не краткосрочный, а долгосрочный характер. Необходимость учета множества трендов, улавливания совокупности «сигналов» со стороны внешнего окружения и адаптации к ним предопределяет новые требования к функциям стратегического планирования и управления и к обеспечению устойчивости осуществления инвестиционных проектов.

Трансформация традиционных подходов к устойчивости проектов. Долгосрочный характер принимаемых решений,

8 World Energy Council [Electronic resource]. URL: https://www.worldenergy.org/ (date accessed: 04.09.2021).

9 Ryser J. Pandemic has accelerated transition to low-carbon economy: Moody's [Electronic resource]. URL: https:// www.spglobal.com/platts/en/market-insights/latest-news/electric-power/102220-pandemic-has-accelerated-transitionto-low-carbon-economy-moodys (date accessed: 11.09.2021).

10 Responsible minerals sourcing for renewable energy [Electronic resource] // Institute for Sustainable Futures. 2019. URL: https://www.earthworks.org/cms/assets/uploads/2019/04/MCEC UTS Report lowres-1.pdf(date accessed: 05.09.2021).

11 The Energy Outlook [Electronic resource] // BP. 2020. 81 p. URL: https://www.bp.com/en/global/corporate/ news-and-insights/press-releases/bp-energy-outlook-2020.html.

12 PBS Newshour, UBS Bank won't fund new offshore Arctic oil, gas projects [Electronic resource]. URL: www. pbs.org/newshour/economy/ubs-bank-wont-fundnew-offshore-arctic-oil-gas-projects (date accessed: 18.09.2021).

13 The 2030 decarbonization challenge. The path to the future of energy [Electronic resource] // Deloitte. $2021.20 \mathrm{p}$. URL: https://www2.deloitte.com/global/en/pages/energy-and-resources/articles/the-2030-decarbonization-challenge.html. 
необходимость учета и адаптация к новым вызовам обуславливают расширение перечня решаемых задач, выходящих за рамки достижения ряда социальных и экологических индикаторов. В таких условиях целесообразно обратиться к возникшему в науке относительно недавно понятию «стратегическая устойчивость инвестиционных проектов», рассматриваемому в российской литературе в контексте деятельности компаний и предприятий в приоритетных отраслях национальной промышленности.

В работе Н.С. Яшина были выделены ключевые подсистемы стратегической устойчивости, к которым относят [21]:

- оперативную устойчивость (способность организации выполнять формируемые планы по выпуску готовой продукции, сведение к минимуму вероятности возникновения «узких мест», выстраивание эффективной производственно-технологической цепочки);

- функциональную устойчивость, включающую в себя маркетинговую, инновационную, производственную, экономическую устойчивость (в соответствии с функциональными процессами);

- рыночную устойчивость (способность к развитию и поддержанию конкурентных преимуществ, нивелирование потенциальных угроз внешней среды и максимизация возможностей).

Можно отметить, что российские авторы не рассматривают стратегическую устойчивость исключительно в плоскости триединства экономических, социальных и экологических параметров, ориентируясь в большей степени на экономические, производственно-технологические и рыночные составляющие. В таблице 1 приведены различные подходы к трактовке понятия «стратегическая устойчивость».

Исходя из приведенных определений понятия стратегической устойчивости, можно условно выделить три ключевых подхода к рассмотрению данной категории: системный, процессный и временной [21]. Их главные различия состоят в направлениях, методах, задачах и целевых приоритетах достижения параметров устойчивости. Так, согласно системному подходу устойчивость компании, проекта или промышленного комплекса может быть достигнута только при условии устойчивого развития подсистем, входящих в их структуру. Исходя из этого, можно сделать вывод, что ключевыми задачами являются:

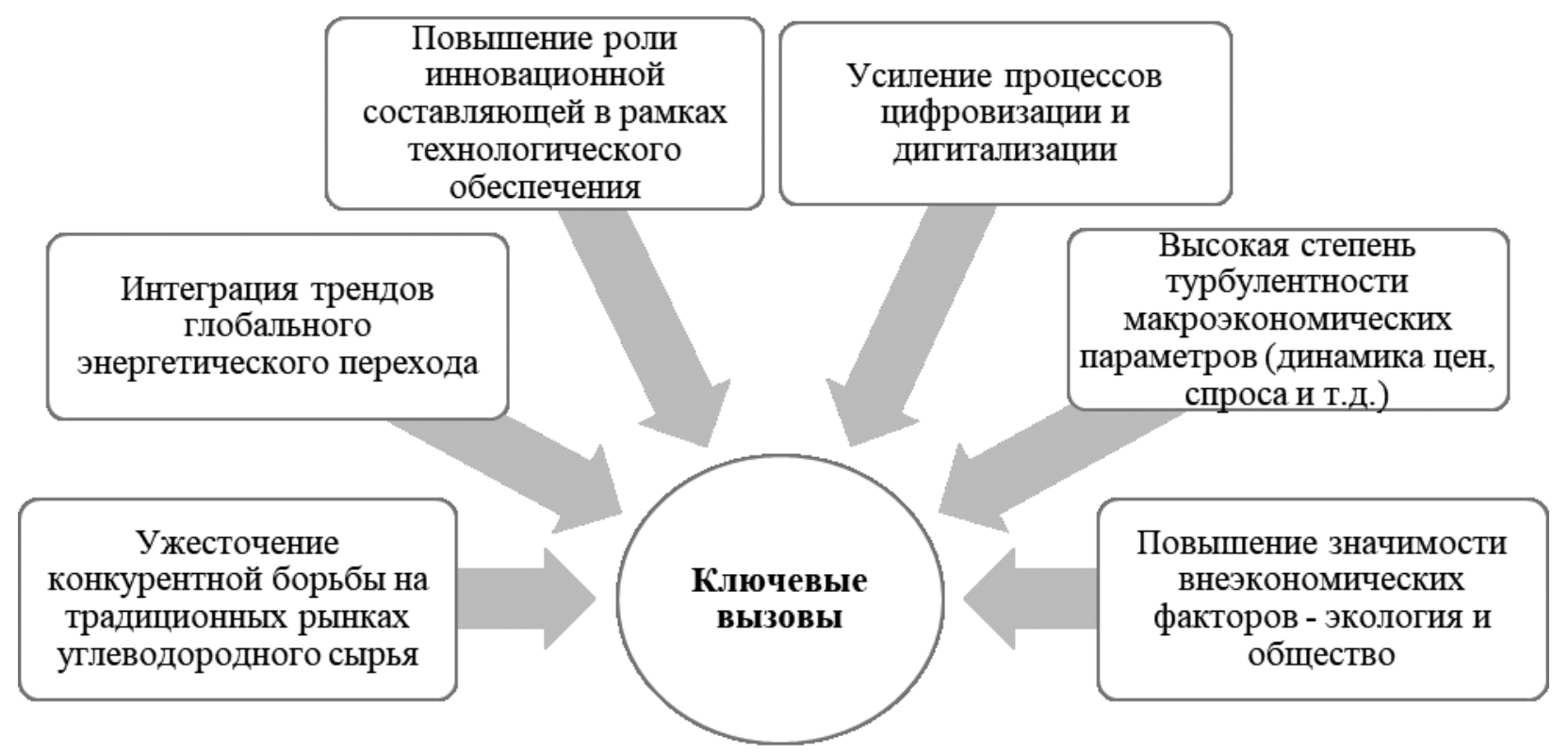

Рис. 3. Систематизация ключевых вызовов энергетического сектора, определяющих высокий уровень турбулентности макроокружения (составлено авторами)

Fig. 3. Systematization of the key challenges of the energy sector that determine the high level of turbulence of the macroenvironment (compiled by the authors) 
Подходы к определению понятия «стратегическая устойчивость» (на основе анализа российских источников литературы) Approaches to the definition of the concept of «strategic sustainability» (based on the analysis of Russian literature sources)

\begin{tabular}{|l|l|}
\hline \multicolumn{1}{|c|}{ Авторы } & \multicolumn{1}{|c|}{ Определение } \\
\hline Н. А. Сабанчиев [14] & $\begin{array}{l}\text { Способность компании (системы, комплекса, проекта) сохранять } \\
\text { свою целостность, достигая поставленных стратегических целей } \\
\text { в постоянно меняющихся условиях внешнего окружения, оказываю- } \\
\text { щих дестабилизирующее воздействие }\end{array}$ \\
\hline М. В. Самосудов [15] & $\begin{array}{l}\text { Способность компании сохранять показатели деятельности в опреде- } \\
\text { ленных пределах на протяжении длительного времени }\end{array}$ \\
\hline Т. В. Терентьева [19] & $\begin{array}{l}\text { Реализация перехода от менее эффективного к более эффективному } \\
\text { состоянию, используя возможности и нивелируя угрозы внешнего } \\
\text { окружения }\end{array}$ \\
\hline С. В. Григорьева [3] & $\begin{array}{l}\text { Сохранение организацией положительной тенденции развития в дол- } \\
\text { госрочном периоде (оценка степени устойчивости осуществляется } \\
\text { на основе комплекса ключевых показателей) }\end{array}$ \\
\hline $\begin{array}{l}\text { В. В. Шеметов, } \\
\text { С.П. Бараненко [1] }\end{array}$ & $\begin{array}{l}\text { Сбалансированность системы управления, организационная ста- } \\
\text { бильность }\end{array}$ \\
\hline $\begin{array}{l}\text { А. Н. Ильченко, } \\
\text { Н.С. Рычихина [13] }\end{array}$ & $\begin{array}{l}\text { Минимизация потерь и нивелирование рисков влияния внешнего ок- } \\
\text { ружения (в случае неблагоприятных условий макроокружения) и ис- } \\
\text { пользование возможностей (при благоприятных макроэкономичес- } \\
\text { ких условиях) }\end{array}$ \\
\hline
\end{tabular}

- сбалансированное развитие отдельных подсистем и элементов комплекса, проекта, организации;

- формирование системы гибкого взаимодействия между подсистемами;

- ориентация на достижение синергетического эффекта взаимодействия отдельных подсистем.

В основе процессного подхода лежит предположение о том, что обеспечение стратегической устойчивости возможно только при условии эффективного управления параметрами внешней и внутренней среды, нивелирования потенциальных угроз и использования имеющихся возможностей. В данном случае приоритетными задачами выступают развитие системы стратегического управления, прогнозирования и планирования, управление факторами риска, ориентация на разработку долгосрочной стратегии развития с учетом микро- и макроэкономических параметров, непрерывный мониторинг новых вызовов и поиск соответствующих решений.

Что касается временного подхода, то стратегическая устойчивость рассматривается в контексте достижения определенного перечня показателей, характеризующих эффективность функционирования исследуемого объекта. Любое отклонение от заданной траектории может быть расценено как потеря стабильности и устойчивости, ввиду чего главные задачи менеджмента состоят в снижении вероятности возникновения рисков, сохранении ключевых параметров деятельности, постоянной корректировке стратегии с целью удержания сформировавшихся позиций.

Каждый из приведенных подходов отражает отдельные аспекты стратегической устойчивости. Для формирования целостного понимания данного понятия необходим ком- 
плексный подход, ведь очевидно, что ориентация на достижение плановых финансовоэкономических показателей не имеет смысла без учета факторов среды, в которой развивается компания (система, комплекс), и принятия мер по обеспечению устойчивости подсистем, входящих в общую структуру.

Объединяя рассмотренные ранее походы, можно выделить ряд принципов, характеризующих стратегическую устойчивость как отдельную категорию [3; 14-15; 29]:

- ориентация на сохранение целостности системы, стабильное функционирование в средне- и долгосрочной перспективе;

- учет факторов конкурентоспособности (нацеленность на наращивание конкурентных преимуществ в долгосрочном периоде);

- учет факторов внешней среды в контексте их влияния на функционирование рассматриваемой системы; решений.

- долгосрочный характер принятия

Устойчивой может быть не только деятельность компании, но и реализация проектов. Согласно наиболее распространенному определению устойчивый проект - это проект, обладающий высокой экономической эффективностью при любых сценариях (негативный, позитивный, реалистичный). Говоря об инвестиционных проектах, выделяют два типа устойчивости: внутренний и внешний.

Внутренний тип характеризуется набором факторов, определяющих возможность достижения эффективности проекта в условиях изменения параметров микро- и макроокружения. Что касается внешней устойчивости проекта, то она определяется стабильностью среды, в которой он непосредственно осуществляется. Согласно современной теории стратегического менеджмента экономические субъекты, будь то проект, компания или комплекс, не только подвержены воздействию внешних факторов, но и способны влиять на них. Данный факт и лежит в основе внешней устойчивости, особенно когда речь идет об осуществлении крупномасштабных проектов.

Считается, что общая устойчивость инвестиционного проекта формируется, исхо- дя из внешней и внутренней устойчивости. Однако здесь возникает планомерный вопрос о том, как оценить степень устойчивости реализации проектов, в том числе при выборе вариантов их осуществления, на какие индикаторы следует опираться и какие методы нужно применять.

Понятие устойчивости проектов также тесно связывают с категорией риска. В целом, исходя из имеющихся подходов к оценке, получается, что стратегическая устойчивость сводится к определению финансовой стабильности и прибыльности проектов в долгосрочной перспективе. Для проведения оценки устойчивости инвестиционных проектов, как правило, используются следующие методы: анализ устойчивости проекта, расчет значений показателей безубыточности проекта, метод вариации параметров проекта, оценка экономической эффективности.

Результаты оцениваются следующим образом: если проект демонстрирует высокие показатели коммерческой эффективности, то он устойчив, в противном случае - нет. Однако такой подход не соответствует самим принципам устойчивого развития. Фокус на финансовые показатели не позволяет оценить другие потенциальные эффекты, возникающие при реализации проектов, особенно если речь идет о масштабных проектах, осуществление которых может привести как к значительным экологическим и социальным последствиям, так и потенциальным мультипликативным эффектам.

Создание единых подходов к оценке устойчивости проектов (компаний, комплексов) представляется сложной задачей, ввиду необходимости учета большого числа параметров, определяющих специфику конкретной отрасли, особенности среды их осуществления и пр.

В Руководстве по оценке проектных предложений Европейского Союза были сформулированы ключевые принципы и критерии устойчивости инвестиционных проектов ${ }^{14}$. Для формирования общего представления о степени устойчивости проекта предлагаются три главных параметра: 1) определение

14 Руководство «Оценка проектных предложений» [Электронный ресурс] // Европейская Комиссия. URL: https://narfu.ru/upload/medialibrary/75e/rukovodstvo-po-otsenke-proektnykh-predlozheniy_es.pdf (дата обращения: 18.09.2021). 


\section{Традиционный подход к устойчивости проектов}

\section{Сущиость}

Стабильность реализации проектов в сфере МСК в условиях изменяющейся внешней и внутренней среды, достижение запланированных индикаторов (в первую очередь, финансово-экономических)

\section{Ключевые направления}

приоритетная роль

планирования индикаторов реализации проекта ориентация на стабильное превышение доходов над расходами рассмотрение стабильности в качестве ключевого фактора (минимизация потерь, избежание вероятных рисков)

Локальный характер формируемых эффектов, главный критерий - финансовая стабильность

\section{Сущность}

Реализация проектов МСК в соответствии с принципами концепции устойчивого развития на основе адаптивности к формирующимся вызовам и открывающимся возможностям путем наращивания конкурентных преимуществ, изменения подходов к стратегическому управлению, следования темпам научно-технического прогресса

\section{Ключевые направления}

разработка гибкой системы индикаторов, ориентированных на оценку экологических, социальных и экологических параметров проектов (с учетом отраслевой специфики)

\section{интеграция основ}

устойчивого развития и $\mathrm{ESG}$ факторов в современную систему стратегического управления, планирования и прогнозирования

\section{взаимоувязка}

инвестиционной привлекательности проектов со степенью ответственности и приверженности принципам УР

Достижение мультипликативных эффектов (на уровне проекта и региона), главный критерий приверженность целям триединства УР (ответственное ведение бизнеса) и адаптивность к условиям турбулентного макроокружения

Рис. 4. Трансформация традиционного подхода к устойчивости проектов в новый подход - стратегическая устойчивость (составлено авторами) Fig. 4. Transformation of the traditional approach to project sustainability into a new approach - strategic sustainability (compiled by the authors) 
уровня воздействия проекта на целевые группы; 2) наличие мультипликативных эффектов при осуществлении проекта; 3) учет наиболее значимых аспектов: политики, экономики, социальной сферы, экологии, технологических параметров и т.д.

Интересен тот факт, что в обозначенном документе инвестиционные проекты рассматриваются как объекты, способные приносить результаты не только в период осуществления, но и после. В частности, особое внимание уделяется тому, будет ли обеспечена безопасность окружающей среды во время и по окончанию реализации проекта (рекультивация, восстановление). Этот факт свидетельствует о тесной взаимосвязи устойчивости и ответственности инициаторов проектов по отношению к окружающей среде, обществу, стейкхолдерам и пр. Такой подход особенно важен для крупномасштабных проектов в сфере промышленности, способных оказать существенное воздействие на экологическую ситуацию в регионах присутствия.

На основе проведенного анализа существующих подходов было сформулировано общее определение стратегической устойчивости проектов, соответствующее комплексному подходу к рассмотрению данной категории. Стратегическая устойчивость проекта - это обеспечение реализации проекта в соответствии с ключевыми принципами концепции устойчивого развития на основе адаптации к формирующимся вызовам и открывающимся возможностям путем наращивания конкурентных преимуществ, изменения подходов к стратегическому управлению, следования темпам научно-технического прогресса.

На рисунке 4 отражены концептуальные основы стратегической устойчивости проекта в рамках формирования комплексного подхода к исследованию данной категории.

Важно заметить, что в условиях высокой степени неопределенности макроокружения и турбулентности внешних параметров критерий «стабильности», используемый многими авторами применительно к устойчивости, не является корректным. В данном случае следует опираться на принцип адаптивности к новым условиям среды, что предполагает совершенствование подходов к стратегическому управлению, планированию и прогнозированию.
Заключение. Таким образом, в рамках проведенного исследования были рассмотрены теоретические основы устойчивого развития и устойчивости применительно к промышленным комплексам, компаниям, проектам. Определена роль УР для компаний, осуществляющих деятельность в горнодобывающем секторе, несмотря на дискуссионный характер обозначенной проблематики.

На примере исследования современных тенденций глобального энергетического сектора обоснован высокий уровень турбулентности и неопределенности макроэкономических условий, характерных для текущего функционирования мировых рынков природных ресурсов. К ключевым вызовам были отнесены такие прогрессивные тренды, как ужесточение конкурентной борьбы на традиционных рынках углеводородного сырья, интеграция трендов глобального энергетического перехода, повышение роли инновационной составляющей в рамках технологического обеспечения, усиление процессов цифровизации и дигитализации, повышение значимости внеэкономических факторов экология и общество.

Установлено, что по мере роста неопределенности макроокружения и интенсификации динамики тенденций на глобальных рынках возникает объективная необходимость пересмотра существующих подходов к УР. Долгосрочный характер принимаемых решений и необходимость адаптации к новым вызовами требованиям (с позиции вклада в ЦУР и соответствия современным требованиям инвесторов) обуславливают расширение перечня решаемых задач, выходящих за рамки достижения ряда социальных и экологических индикаторов и предопределяющих необходимость перехода от классического УР к стратегической устойчивости проектов, ориентированной на обеспечение реализации проекта в соответствии с ключевыми принципами концепции устойчивого развития на основе адаптации к формирующимся вызовам и открывающимся возможностям путем наращивания конкурентных преимуществ, изменения подходов к стратегическому управлению, следования темпам научнотехнического прогресса.

В исследовании разработаны концептуальные основы стратегической устойчивости 
проекта, соответствующие новому подходу к оценке УР и основанные на учете неопределенности и высокого уровня турбулентности макроокружения.

\section{Список источников}

1. Бараненко С.П., Шеметов В.В. Стратегическая устойчивость предприятия. М.: Полиграф, 2004. 492 с.

2. Бирюкова В.В., Череповицын А.Е. Российский и зарубежный опыт формирования индексов устойчивого развития вертикально-интегрированных нефтяных компаний // Экономика и управление: научно-практический журнал. 2020. №6(156). С. 5-10.

3. Григорьева С.В. Оценка стратегической устойчивости в развитии предприятия// Вопросы экономики и права. 2013. №3. С. 33-37.

4. Доклад о Целях в области устойчивого развития. Организация Объединенных Наций. $2020.68 \mathrm{c}$.

5. Ильинова А.А., Соловьева В.М. Стратегическое планирование и прогнозирование: изменение сущности и роли в условиях нестабильности энергетического сектора // Север и рынок: формирование экономического порядка. 2021. №2(72). С. 56-68.

6. Коробейникова А.К., Невская М.А. Проблемы цифровой трансформации в современных условиях // Сборник трудов национальной научно-практической конференции с международным участием «Цифровые технологии в экономике и промышленности». Под редакцией А. В. Бабкина. СПб.: ПолитехПресс. 2019. С. 185-192.

7. Митрова Т., Грушевенко Е., Капитонов С., Мельников Ю., Пердеро А., Доброславский Н. Коронакризис: влияние COVID-19 на ТЭК в мире и в России [Электронный pecypc]. М.: Московская школа управления Сколково, 2020. URL: https://mks-group.ru/ storage/presentations/SKOLKOVO_EneC COVID19_and_Energy_sector_RU.pdf- (дата обращения: 09.09.2021).

8. Невская М.А., Маринина О. А. Стимулирование инновационных преобразований в целях устойчивого развития минеральносырьевого сектора России // Интернет-журнал Науковедение. 2017. Т. 9. №6. С. 33.

9. Основная цель развития до 2030 года: успешный бизнес и стабильное будущее.
Взаимосвязь устойчивого развития и долгосрочного коммерческого успеха [Электронный ресурс] // Deloitte, январь 2017. 72 с. URL: https://gog.greenmobility.ru/UserFiles/ Files/sustainable-development-goals-2030.pdf.

10. Пикалова Т.А. Алгоритм целевого управления операционной деятельностью горнодобывающей компании в контексте устойчивого развития // Горный информационно-аналитический бюллетень (научно-технический журнал). 2014. №2. С. 380-386.

11. Пономаренко Т. В., Сидоров Д.В., Ларичкин Ф. Д., Новосельцева В.Д. Экономическая устойчивость горно-металлургических компаний при реализации инвестиционных проектов // Рациональное освоение недр. 2020. №5. С. 54-63.

12. Пронин Э.М., Васильев В.Е., Цветков В.Ю. Факторы, определяющие устойчивое развитие предприятий минерально-сырьевого комплекса, и их влияние на оценку результатов деятельности предприятий // Записки Горного Института. 2011. №191. С. 176.

13. Рычихина Н.С. Реструктуризация как инструмент достижения стратегической устойчивости предприятия // Экономический анализ: теория и практика. 2008. №10(115). C. 57-62.

14. Сабанчиев Н.А. Гибкость, управляемость и стратегическая устойчивость: понятия, взаимосвязь и оценка // Российский экономический Интернет-журнал. 2012. №1. C. 803-813.

15. Самосудов М.В. Корпоративное управление: Теория корпоративного взаимодействия. Модульная серия «Экономистмеждународник». М.: Всероссийская академия внешней торговли Минэкономразвития России, 2006. С. 330.

16. Сергеев И. Б., Лапочкина Л. В. Устойчивое развитие: теоретико-методологические подходы // Записки Горного Института. 2009. №184. C. 264.

17. Сысоева Е.Ф. Структура капитала и финансовая устойчивость организации: практический аспект // Финансы и кредит. 2007. №22. C. 24-29.

18. Тенденции развития - 2021 [Электронный ресурс] // Deloitte, 2021. 94 c. URL: https://www2.deloitte.com/content/dam/Deloitte/ $\mathrm{ru} /$ Documents/energy-resources/Russian/RU_ Tracking-the-trends-2021_final.pdf. 
Вестник ЮРГТУ (НПИ). Серия: Сочиально-экономические науки. 2021 г. T. 14. № 6 Bulletin of the SRSTU (NPI). Series: Socio-Economic Sciences. 2021. Vol. 14. № 6

19. Терентьева Т.В., Бобырева М.А. Устойчивое развитие предпринимательской структуры как категория. Концептуальные подходы к трактовке категории «устойчивость развития» предпринимательской структуры // Российское предпринимательство. 2011. №1-2. C. 52-56.

20. Фоломьев А.Н. Устойчивость предприятий в рыночном хозяйстве. М.: Прогресс, 1995. 245 с.

21. Яшин Н. С., Григорян Е. С. Методология стратегической устойчивости предприятия // Вестник Саратовского государственного социально-экономического университета. 2015. C. 18-22.

22. Ansoff H.I., McDonnell E. Implanting Strategic Management, 2ed.; Prentice-Hall: New York, NY, USA, 1990. 515 p.

23. Church C., Crawford A. Minerals and the Metals for the Energy Transition: Exploring the Conflict Implications for Mineral-Rich, Fragile States The Geopolitics of the Global Energy Transition // Lecture Notes in Energy. 2020. №73. Pp. 279-304.

24. Dawn of a new era [Electronic resource]// Oil and gas price benchmarking as at 30 June 2020. URL: https://www.pwc.co.uk/oilgas/assets/oil-gas-commodity-price-benchmarking.pdf (date accessed: 05.09.2021).

25. Diesendorf M. Sustainability and sustainable development // Sustainability: The corporate challenge of the 21st century, Sydney: Allen\&Unwin. 2000. Chap. 2. Pp. 19-37.

26. DNV GL Energy transition outlook [Electronic resource]. URL: DNV GL Energy_Transition_Outlook_2020_main_report lowres_single.pdf (date accessed: 15.09 .2021 ).

27. ESG факторы в инвестировании [Электронный ресурс] // PWC. МИРБИС, 2019. URL: https://www.pwc.ru/ru/sustainability/assets/pwc-responsible-investment.pdf (дата обращения: 29.09.2021).

28. Holden E., Linnerud K., Banister D. Sustainable development: Our common future revisited // Global Environmental Change. 2014. №26. Pp. 130-139.

29. Huckle J. Education for sustainable development in Europe: Some current challenges. Presentation to a conference on environmental education organised by the Hellenic Association for the Protection of the Environment and Cultural Heritage // University of Piraeus, 2005.
30. Ruggerio C.A. Sustainability and sustainable development: A review of principles and definitions // Science of the Total Environment. 2021. 786. 147481.

31. Tsvetkova A., Katysheva E. Ecological and economic efficiency evaluation of sustainable use of mineral raw materials in modern conditions // International Multidisciplinary Scientific GeoConference Surveying Geology and Mining Ecology Management, SGEM. 2017. №217(53). Pp. 259-266.

\section{References}

1. Baranenko S.P., Shemetov V.V. Strategicheskaja ustojchivost' predprijatija [Strategic stability of the enterprise]. Moscow: Poligraf, 2004. 492 p. (In Russ.).

2. Birjukova V. V., Cherepovicyn A. E. Rossijskij i zarubezhnyj opyt formirovanija indeksov ustojchivogo razvitija vertikal'no-integrirovannyh neftjanyh kompanij [Russian and foreign experience in the formation of indices of sustainable development of vertically integrated oil companies]. Jekonomika i upravlenie: nauchnoprakticheskij zhurnal [Economics and Management: a scientific and practical journal]. 2020; 6(156): 5-10. (In Russ.).

3. Grigor'eva S.V. Ocenka strategicheskoj ustojchivosti v razvitii predprijatija [Assessment of strategic sustainability in the development of the enterprise]. Voprosy jekonomiki i prava [Issues of economics and law]. 2013; (3): 33-37. (In Russ.).

4. Doklad o Celjah v oblasti ustojchivogo razvitija. Organizacija Ob'edinennyh Nacij [Report on the Sustainable Development Goals. United Nations]. 2020. 68 p. (In Russ.).

5. Il'inova A. A., Solov'eva V.M. Strategicheskoe planirovanie i prognozirovanie: izmenenie sushhnosti i roli v uslovijah nestabil'nosti jenergeticheskogo sektora [Strategic planning and forecasting: changing the essence and role in the conditions of instability of the energy sector]. Sever i rynok: formirovanie jekonomicheskogo porjadka [The North and the market: the formation of an economic order]. 2021; 2(72): 56-68. (In Russ.).

6. Korobejnikova A.K., Nevskaja M.A. Problemy cifrovoj transformacii $v$ sovremennyh uslovijah [Problems of digital transformation in modern conditions]. Sbornik trudov nacional'noj nauch- 
no-prakticheskoj konferencii s mezhdunarodnym uchastiem «Cifrovye tehnologii $\mathrm{v}$ jekonomike $\mathrm{i}$ promyshlennosti». Pod redakciej A.V. Babkina [Proceedings of the national scientific and practical conference with international participation «Digital technologies in economics and industry». In A.V. Babkin (eds.)]. Saint Petersburg: PolitehPress. 2019. Pp. 185-192. (In Russ.).

7. Mitrova T., Grushevenko E., Kapitonov S., Mel'nikov Ju., Perdero A., Dobroslavskij N. Koronakrizis: vlijanie COVID-19 na TJeK v mire i v Rossii [Coronaries: the impact of COVID-19 on the energy sector in the world and in Russia] [Jelektronnyj resurs]. Moscow: Moskovskaja shkola upravlenija Skolkovo, 2020. URL: https://mksgroup.ru/storage/presentations/SKOLKOVO EneC_COVID19_and_Energy_sector_RU.pdf (date accessed: 09.09.2021). (In Russ.).

8. Nevskaja M.A., Marinina O.A. Stimulirovanie innovacionnyh preobrazovanij $\mathrm{v}$ celjah ustojchivogo razvitija mineral'no-syr'evogo sektora Rossii [Incentives for innovation and transformation in the sustainable development of the mineral resources sector of Russia]. Internetzhurnal Naukovedenie [Online journal of Science Studies]. 2017; 9(6): 33. (In Russ.).

9. Osnovnaja cel' razvitija do 2030 goda: uspeshnyj biznes i stabil'noe budushhee. Vzaimosvjaz' ustojchivogo razvitija i dolgosrochnogo kommercheskogo uspeha [The main goal of development until 2030: a successful business and a stable future. The relationship of sustainable development and long-term commercial success] [Jelektronnyj resurs]. Deloitte, janvar' 2017. 72p. URL: https://gog.greenmobility.ru/UserFiles/Files/sustainable-developmentgoals-2030.pdf. (In Russ.).

10. Pikalova T.A. Algoritm celevogo upravlenija operacionnoj dejatel'nost'ju gornodobyvajushhej kompanii v kontekste ustojchivogo razvitija [Algorithm of target management of operational activities of a mining company in the context of sustainable development]. Gornyj informacionno-analiticheskij bjulleten' (nauchno-tehnicheskij zhurnal) [Mining information and analytical bulletin (scientific and technical journal)]. 2014; (2): 380-386. (In Russ.).

11. Ponomarenko T. V., Sidorov D. V., Larichkin F.D., Novosel'ceva V.D. Jekonomicheskaja ustojchivost' gorno-metallurgicheskih kompanij pri realizacii investicionnyh proektov [Economic sustainability of mining and metallurgical companies in the implementation of investment projects]. Racional'noe osvoenie nedr [Rational development of mineral resources]. 2020; (5): 54-63. (In Russ.).

12. Pronin Je. M., Vasil'ev V. E., Cvetkov V. Ju. Faktory, opredeljajushhie ustojchivoe razvitie predprijatij mineral'no-syr'evogo kompleksa, i ih vlijanie na ocenku rezul'tatov dejatel'nosti predprijatij [Determinants of sustainable development of enterprises of the mineral complex, and their impact on the performance of enterprises]. Zapiski Gornogo Instituta [Notes of the Mining Institute]. 2011; (191): 176. (In Russ.).

13. Rychihina N.S. Restrukturizacija kak instrument dostizhenija strategicheskoj ustojchivosti predprijatija [Restructuring as a tool for achieving strategic sustainability of the enterprise]. Jekonomicheskij analiz: teorija i praktika [Economic analysis: theory and practice]. 2008; 10(115): 57-62. (In Russ.).

14. Sabanchiev N.A. Gibkost', upravljaemost' i strategicheskaja ustojchivost': ponjatija, vzaimosvjaz' i ocenka [Flexibility, manageability and strategic stability: concepts, interrelation and evaluation]. Rossijskij jekonomicheskij Internet-zhurnal [Russian Economic Online Journal]. 2012; (1): 803-813. (In Russ.).

15. Samosudov M. V. Korporativnoe upravlenie: Teorija korporativnogo vzaimodejstvija. Modul'naja serija «Jekonomist-mezhdunarodnik» [Corporate governance: Theory of corporate interaction. Modular series «International Economist»]. Moscow: Vserossijskaja akademija vneshnej torgovli Minjekonomrazvitija Rossii, 2006. P. 330. (In Russ.).

16. Sergeev I. B., Lapochkina L. V. Ustojchivoe razvitie: teoretiko-metodologicheskie podhody [Sustainable development: theoretical and methodological approaches]. Zapiski Gornogo Instituta [Notes of the Mining Institute]. 2009; (184): 264. (In Russ.).

17. Sysoeva E. F. Struktura kapitala i finansovaja ustojchivost' organizacii: prakticheskij aspekt [Capital structure and financial stability of an organization: a practical aspect]. Finansy $i$ kredit [Finance and credit]. 2007; (22): 24-29. (In Russ.).

18. Tendencii razvitija - 2021 [Development trends - 2021] [Jelektronnyj resurs]. Deloitte, 2021. 94 p. URL: https://www2. deloitte.com/content/dam/Deloitte/ru/Docu- 
Вестник ЮРГТУ (НПИ). Серия: Сочиально-экономические науки. 2021 г. T. 14. № 6 Bulletin of the SRSTU (NPI). Series: Socio-Economic Sciences. 2021. Vol. 14. № 6

ments/energy-resources/Russian/RU_Trackingthe-trends-2021_final.pdf. (In Russ.).

19. Terent'eva T. V., Bobyreva M.A. Ustojchivoe razvitie predprinimatel'skoj struktury kak kategorija. Konceptual'nye podhody $\mathrm{k}$ traktovke kategorii «ustojchivost' razvitija» predprinimatel'skoj struktury [Sustainable development of the entrepreneurial structure as a category. Conceptual approaches to the interpretation of the category "sustainability of development» of an entrepreneurial structure]. Rossijskoe predprinimatel'stvo [Russian entrepreneurship]. 2011; (1-2): 52-56. (In Russ.).

20. Folom'ev A.N. Ustojchivost' predprijatij $\mathrm{v}$ rynochnom hozjajstve [Sustainability of enterprises in the market economy]. Moscow: Progress, 1995. 245 p. (In Russ.).

21. Jashin N.S., Grigorjan E.S. Metodologija strategicheskoj ustojchivosti predprijatija [Methodology of strategic sustainability of the enterprise]. Vestnik Saratovskogo gosudarstvennogo social'no-jekonomicheskogo universiteta [Bulletin of the Saratov State Socio-Economic University]. 2015; 18-22. (In Russ.).

22. Ansoff H.I., McDonnell E. Implanting Strategic Management, 2ed.; Prentice-Hall: New York, NY, USA, 1990. 515 p.

23. Church C., Crawford A. Minerals and the Metals for the Energy Transition: Exploring the Conflict Implications for Mineral-Rich, Fragile States The Geopolitics of the Global Energy Transition // Lecture Notes in Energy. 2020. №73. Pp. 279-304.

24. Dawn of a new era [Electronic resource]// Oil and gas price benchmarking as at 30 June 2020. URL: https://www.pwc.co.uk/oil- gas/assets/oil-gas-commodity-price-benchmarking.pdf (date accessed: 05.09.2021).

25. Diesendorf M. Sustainability and sustainable development // Sustainability: The corporate challenge of the 21st century, Sydney: Allen\&Unwin. 2000. Chap. 2. Pp. 19-37.

26. DNV GL Energy transition outlook [Electronic resource]. URL: DNV_GL_Energy_Transition_Outlook_2020_main_report lowres_single.pdf (date accessed: 15.09 .2021 ).

27. ESG факторы в инвестировании [Электронный ресурс] // PWC. МИРБИС, 2019. URL: https://www.pwc.ru/ru/sustainability/assets/pwc-responsible-investment.pdf (дата обращения: 29.09.2021).

28. Holden E., Linnerud K., Banister D. Sustainable development: Our common future revisited // Global Environmental Change. 2014. №26. Pp. 130-139.

29. Huckle J. Education for sustainable development in Europe: Some current challenges. Presentation to a conference on environmental education organised by the Hellenic Association for the Protection of the Environment and Cultural Heritage // University of Piraeus, 2005.

30. Ruggerio C.A. Sustainability and sustainable development: A review of principles and definitions // Science of the Total Environment. 2021. 786. 147481.

31. Tsvetkova A., Katysheva E. Ecological and economic efficiency evaluation of sustainable use of mineral raw materials in modern conditions // International Multidisciplinary Scientific GeoConference Surveying Geology and Mining Ecology Management, SGEM. 2017. №217(53). Pp. 259-266.

Статья поступила в редакцию 05.10.2021; одобрена после рещензирования 25.10.2021; принята к публикачии 18.11.2021.

The article was submitted on 05.10.2021; approved after reviewing on 25.10.2021; accepted for publication on 18.11.2021. 


\section{ИНФОРМАЦИЯ ОБ АВТОРАХ}

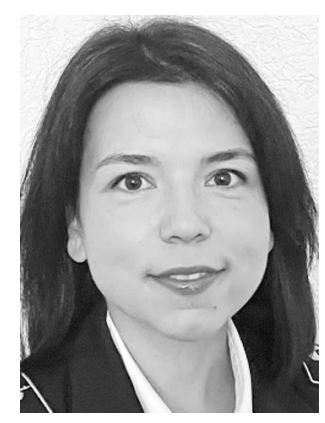

Дмитриева Диана Михайловна - кандидат экономических наук, доцент кафедры «Экономика, организация и управление», Санкт-Петербургский горный университет.

Россия, г. Санкт-Петербург, Васильевский остров, 21 линия, 2

Diana M. Dmitrieva - Candidate of Economic Sciences, Associate Professor, Department of Economics, Organization and Management, Saint-Petersburg Mining University.

221st Liniya st., Saint-Petersburg, Russia

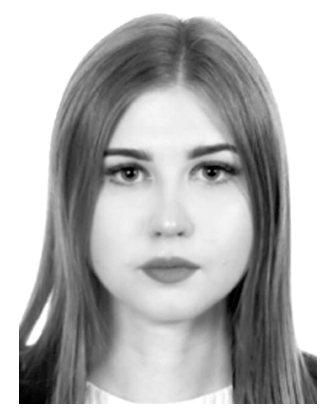

Соловьева Виктория Максимовна - аспирант кафедры «Экономика, организация и управление» экономического факультета, Санкт-Петербургский горный университет.

Россия, г. Санкт-Петербург, Васильевский остров, 21 линия, 2

Victoria M. Solovyova - Postgraduate Student, Department of Economics, Organization and Management, Saint Petersburg Mining University.

2 21st Liniya st., Saint-Petersburg, Russia

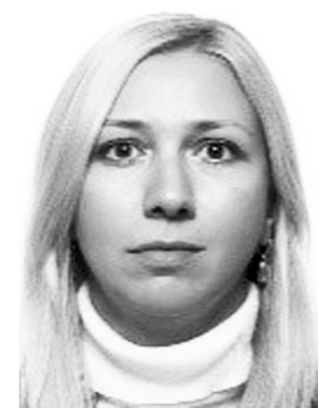

Рутенко Евгения Григорьевна - аспирант, кафедра «Экономика, организация и управление», Санкт-Петербургский горный университет.

Россия, г. Санкт-Петербург, Васильевский остров, 21 линия, 2

Evgeniya G. Rutenko - Postgraduate Student, Department of Economics, Organization and Management, Saint-Petersburg Mining University.

221st Liniya st., Saint-Petersburg, Russia

\section{Вклад авторов:}

Дмитриева Д. М. - научное руководство; концепция исследования; развитие методологии; итоговые выводы.

Соловьева В. М. - написание исходного текста; итоговые выводы.

Рутенко Е.Г. - информационно-аналитическое обеспечение; доработка текста.

Contribution of the authors:

Dmitrieva D. M. - scientific management; research concept; development of methodology; final conclusions.

Solovyova V.M. - writing the source text; final conclusions.

Rutenko E. G. - information and analytical support; follow-on version of the text. 